\title{
Optical Fibre Technology
}

\author{
Gerald Farrell \\ Photonics Research Centre, School of Electronic and Communications Engineering, \\ Dublin Institute of Technology, Kevin Street, Dublin 8, Ireland
}

Received December 29, 2012; accepted December 30, 2012; published December 31, 2012

\begin{abstract}
A brief editorial overview of the papers devoted to optical fibre technology in this special edition of Photonics Letters of Poland.
\end{abstract}

Since the first demonstrations of systems in the 1970s, optical fibre technology has seen continuous development and an ever broadening range of applications. Optical fibre communication systems are now the premier technology for very high bit rate communications over national and global distances, underpinning the Internet and new entertainment technologies such as on-line gaming and on-demand television. Optical fibre based sensing has also developed rapidly to a point where it is the technology of choice for many applications, from the micro world of bio-sensing to the macro world of sensorised smart composites for aeronautical and other engineering applications.

New challenges are continually emerging for optical fibre technology and this special edition describes a variety of recent developments in optical fibre technology.

The need for ever greater capacity in national communication backbones and other optical networks is a key challenge. A paper on generation and reception at bit rates up to $28 \mathrm{Gbit} / \mathrm{s}$ demonstrates the useful result that such transmission rates are possible with more economical limited-bandwidth components.

Tapered fibres and fibre Bragg gratings (FBGs) each have a wide range of useful optical properties. Combining the two together is a challenge but in a paper presented here the authors propose an approach to shaping the spectral characteristics of fibre Bragg gratings written in an optical fibre taper using a phase mask method.

Slow light is a phenomenon that has received a lot of attention in recent years, mainly as the basis for simple delay-based data buffers. It can also be applied to sensing and a paper presented here demonstrates how to enhance the sensitivity of an interferometer by utilising slow light in photonic crystal waveguide.

Polarisation maintaining fibre (PMF) has been used in a variety of sensing applications to date. A paper is presented which describes a Microfibre Sagnac Interferometer that utilises a section of PMF containing a tapered portion. The result is enhanced sensitivity for refractive index sensing with low temperature dependence.
Microstructured optical fibre (MOF) has received a lot of attention in recent years. In a paper in this edition the properties of an all-solid microstructured optical fibre are investigated. Such fibres allow one to utilise the useful properties of an MOF but without the difficulties of microhole collapse during the fabrication of air-hole MOFs

Integrated optical devices are a key element of many modern optical fibre systems. Ion exchange processes are used in planar waveguide fabrication and a paper here investigates the degree of permeability of silica layers, produced by the sol-gel method, used as masks for ionexchange processes.

Optical fibre based light sources have developed significantly in the last few years both in terms of performance and diversity of types. In a paper here the authors report on the development of a $2.0 \mathrm{~mW}$ Er-doped C-band Super fluorescent Fibre Source pumped at 980 $\mathrm{nm}$, for applications in fibre sensing in which a broad spectral band and a high power source are required.

Chalcogenide glasses offer a wealth of attractive properties such as exceptionally high nonlinearity, and an ultrafast nonlinear response. Singlemode-MultimodeSinglemode (SMS) optical fibre structures have proved to be very useful for sensing and other applications. A paper is presented on an SMS structure utilising a chalcogenide multimode fibre, as a basis for the development of compact and robust photonic nonlinear devices.

Another paper describes the studies of a wideband erbium doped fiber amplifier which utilizes a tunable tap at the source end. It is shown that a gain higher than 20 $\mathrm{dB}$ is achievable over the $\mathrm{C}$ and $\mathrm{L}$ bands from $1525-1615$ $\mathrm{nm}$.

A number of more general papers are also included in this edition, covering areas such as gas detection using an infared imaging Fourier-transform spectrometer and the spectroscopic properties of rare earth ions in tellurite glass.

In closing, I want to thank all the authors for their contributions to this special edition. I also want to thank Dr. Katarzyna Rutkowska and Prof. Tomasz Woliński for their help in preparing this special edition of Photonics Letters of Poland. 